\section{BMJ Open Respiratory Research}

\title{
Fever is associated with higher morbidity and clot burden in patients with acute pulmonary embolism
}

Muhammad Saad, ${ }^{1,2}$ Danial H Shaikh, ${ }^{1,2}$ Nikhitha Mantri, ${ }^{1,2}$ Ahmed Alemam, ${ }^{1,2}$ Aiyi Zhang, ${ }^{1,2}$ Muhammad Adrish ${ }^{1,2}$

\section{ABSTRACT}

Background Fever is considered as a presenting symptom of pulmonary embolism (PE). We aim to evaluate the association between PE and fever, its clinical characteristics, outcomes and role in prognosis. Methods A retrospective chart review of patients who were hospitalised with the diagnosis of acute PE was conducted. Patients in whom underlying fever could also be attributable to an underlying infection were also excluded.

Results A total of 241 patients met the study criteria. 63 patients (25.7\%) had fever within 1 week of diagnosis of PE of which four patients had fever that could be due to underlying infection and were excluded. Patients in PE with fever group were younger compared with PE without fever group (52.52 vs $58.68, \mathrm{p}=0.012$ ) and had higher incidence of smoking ( $44.1 \%$ vs $20.9 \%, p<0.001)$. Patients in PE with fever group were more likely to require intensive care admission ( $69.5 \%$ vs $35.7 \%, p<0.001)$, had a longer hospital length of stay $(19.80 \mathrm{vs} 12.20, p<0.001)$ and higher requirement of mechanical ventilation $(30.5 \%$ vs $6.6 \%, p<0.001)$ compared with those without fever. PE with fever group were more likely to have massive and submassive PE (55.9\% vs $36.8 \%, p=0.015)$ and had higher incidence of deep vein thrombosis ( $33.3 \%$ vs $17.4 \%$, $\mathrm{p}=0.0347$ ) compared with PE without fever. In a univariate model, there was higher likelihood of in-hospital mortality in PE with fever group compared with PE without fever ( $22.0 \%$ vs $10.4 \%, p=0.039$ ).

Conclusion Patients with acute PE and fever have higher morbidity and clot burden.

Check for updates

(c) Author(s) (or their employer(s)) 2018. Re-use permitted under CC BY-NC. No commercial re-use. See rights and permissions. Published by BMJ.

${ }^{1}$ Department of Internal Medicine, BronxCare Health System, Bronx, New York, USA

${ }^{2}$ Department of Medicine, Affiliated with Icahn School of Medicine at Mt Sinai, New York, USA

Correspondence to Dr Muhammad Adrish; aadrish@hotmail.com

\section{INTRODUCTION}

Pulmonary embolism (PE) is among the leading causes of hospital-associated complications, preventable hospital deaths and healthcare costs. ${ }^{1}$ Attributable death rate in these patients ranges up to $10 \%-30 \%$ (if untreated) and $8 \%$ (if treated) in hospitalised population with economic burden exceeding 1.5 billion dollars in USA. ${ }^{23}$ According to the regional experience in several US studies, PE has an incidence of approximately 71-117 per 100000 person-years ${ }^{4}$ with prevalence as high as $2 \% .{ }^{5}$ In a community-based study by radiologists using CT angiogram, PE was diagnosed

\section{Key messages}

Significance of fever in patients with acute pulmonary embolism.

- Fever may signify higher clot burden and poor outcomes.

- How we came to this conclusion and what is the current evidence.

in $13 \%$ of hospitalised patients $(132 / 981){ }^{6}$ To evaluate the dynamics of this menace of death, several studies have been performed and reported various aspects of diagnostic, therapeutic and prognostic strategies but data remain limited regarding the symptomatic accuracy of the disease. ${ }^{78}$ Although cough, pleuritic chest pain and dyspnoea have been mentioned as the most common symptoms of PE, fever has been considered a surrogate symptom and sought with interest as a cause of physician's apprehension, unnecessary use of antibiotics and diagnostic evaluation. ${ }^{9}$

Roughly half of all patients with PE and deep vein thrombosis (DVT) are noted to have fever ranging from $38^{\circ} \mathrm{C}$ to $38.5^{\circ} \mathrm{C} .{ }^{10}{ }^{11}$ In the landmark PIOPED study, ${ }^{12}$ fever (temperature at or above $37.8^{\circ} \mathrm{C}$ ) was observed in $14 \%$ patients with no identifiable cause. Similarly, PISAPED study ${ }^{13}$ demonstrated fever above $38^{\circ} \mathrm{C}$ in 6 out of 440 patients with PE. Murray et al noted temperature of $\geq 38^{\circ} \mathrm{C}\left(\geq 100.4^{\circ} \mathrm{F}\right)$ in $64 \%$ and $6 \%$ with temperature of $\geq 39.5^{\circ} \mathrm{C}$ $\left(\geq 103.1^{\circ} \mathrm{F}\right)$ in patients with PE. ${ }^{14}$ Israel and Goldstein indicated low-grade fever in $78.9 \%$ of patients despite the use of antibiotics. ${ }^{15} \mathrm{On}$ contrary, in a prediction model for the probability of PE proposed by Miniati et al, fever was not considered a major predictor of PE, but the study did not receive appropriate external validity. ${ }^{16}$ Later, in a study by Kokturk et al, 33\% patients with PE developed fever, of which $12 \%(5 / 39)$ subjects had temperature over $39^{\circ} \mathrm{C} .{ }^{17}$ 
Despite all efforts exercised to identify role of fever as a diagnostic predictor of $\mathrm{PE}$, less is known about its prognostic role and importance in management algorithm. ${ }^{18}$ We intend to identify the association between $\mathrm{PE}$ and fever, clinical characteristics of patients with PE presenting with fever and its prognostic implications, if any.

\section{METHODS AND MATERIAL}

\section{Study design}

A retrospective chart review of patients with newly diagnosed acute PE admitted from 1 January 2008 to 31 January 2018 to BronxCare Health System was conducted. A total of 530 patients were identified, of which 285 histories of previously diagnosed acute PE were excluded. A total of 245 patients had acute PE diagnosed during the hospital stay of which 63 patients (25.7\%) had fever within 1 week of diagnosis of PE. Of these 63 patients, 4 had fever that may have been due to an underlying infection and therefore were excluded. A total of 241 patients were included in final analysis (figure 1). We classified our patients into two groups, namely PE with fever and PE without fever. We collected data regarding patient's baseline demographics, comorbid conditions, vital signs, laboratory parameters and severity of $\mathrm{PE}$. Cultures and radiographic data within 1 week of fever were also collected to assess for infection as underlying source of fever. We further gathered data regarding use of anticoagulation, antibiotics and tissue plasminogen activator. Primary outcome in our study was mortality difference between the two groups. Secondary outcomes were severity of PE, length of stay (LOS) in hospital, need for intensive care unit (ICU) admission, need for mechanical ventilation.

Study was approved by the institutional review board (IRB\#01111803).

\section{DEFINITIONS}

Following definitions were used in our study:

- Fever was defined as temperature of $\geq 100.4^{\circ} \mathrm{F}$ within 1 week of diagnosis of PE. All cultures and radiographic

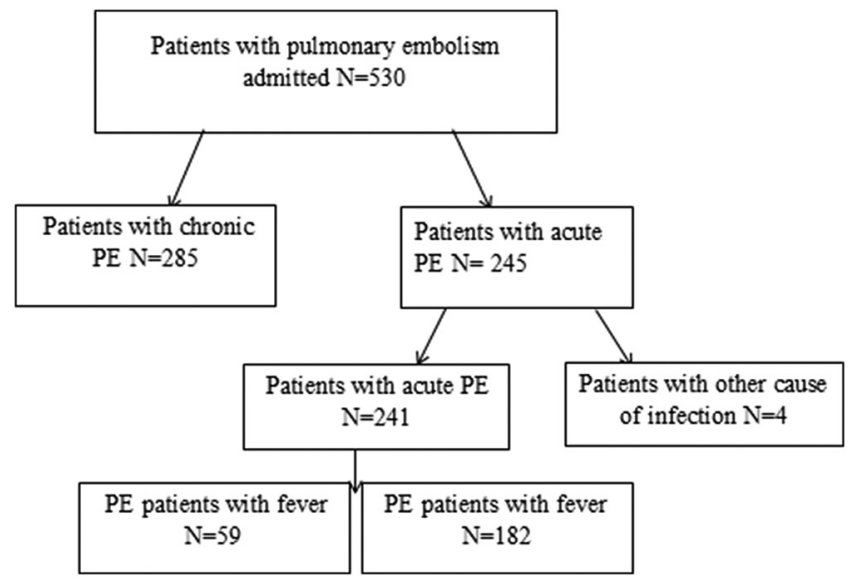

Figure 1 Schematic picture of study design. PE, pulmonary embolism. data were reviewed and patients with evidence of an active infection or those in whom an underlying infection could not be excluded with certainty were removed from the final analysis. We excluded four such patients of which two died during the hospital stay. Furthermore, there were four patients with positive cultures who were included in the study as those cultures were deemed colonisers.

- Massive PE was defined as PE with sustained hypotension (systolic blood pressure $<90 \mathrm{~mm} \mathrm{Hg}$ ). ${ }^{12}$

- Submassive PE was defined patients with systolic blood pressure $>90 \mathrm{~mm} \mathrm{Hg}$ but evidence of right ventricular dysfunction on echocardiograms or on the CT pulmonary angiograms or evidence of myocardial damage as reflected by increase in cardiac biomarkers. ${ }^{12} 14$

\section{STATISTICAL ANALYSIS}

Demographic information and clinical outcomes were stratified by fever status. Means and SD were reported for continuous variables. Frequencies and percentages were reported for categorical variables. ANOVA tests were used to assess the association between continuous variables and fever status. $\chi^{2}$ tests were used to assess the association between categorical variables and fever status. For the relationship between ventilation usage and mortality, subgroup analyses were conducted by antibiotic usage status. Frequencies and percentages were reported. $\chi^{2}$ tests were used to assess the association between the outcome variables and fever status. Separate multivariable logistic regressions were used to assess the association between fever status and if the patient is expired or not, need for mechanical ventilation, need for ICU admission, controlling age, pulmonary infarction, heart rate, systolic blood pressure, leucocyte count, congestive heart failure (CHF), smoking history and if the patient had simple or submassive and massive PE. Multivariable linear regression was used to assess the association between fever status and LOS controlling age, pulmonary infarction, heart rate, systolic blood pressure, leucocyte count, CHF, smoking history and if the patient had simple or submassive and massive PE.

\section{RESULTS}

Table 1 indicates demographic and clinical information stratified by fever status. There were 241 patients in the study. Patients in PE with fever group were younger compared with those without fever (52.52 vs $58.68, \mathrm{p}=0.012)$, had higher heart rate $(101.14$, vs 90.20 , $\mathrm{p}<0.001)$, had lower mean systolic blood pressure (115.93 vs $123.72, \mathrm{p}=0.013$ ) and higher mean leucocyte count (10.98 vs 9.80, $\mathrm{p}=0.006)$. Among patients with fever, the percentage of antibiotic users were higher than the percentage of non-antibiotic users $(62.7 \%$ vs $26.9 \%$, $\mathrm{p}<0.001)$. During evaluation of underlying comorbid conditions, we found that patients with fever and PE compared with those without fever had higher incidence 
Table 1 Demographic and clinical variables

\begin{tabular}{|c|c|c|c|c|}
\hline Variables & PE with fever $(n=59)$ & $\begin{array}{l}\text { PE without fever } \\
(n=182)\end{array}$ & Total $(n=241)$ & $P$ values \\
\hline Age & $52.518(15.457)$ & $58.678(16.452)$ & 57.170 (16.399) & 0.01 \\
\hline Gender (Female) & $27(45.8 \%)$ & $94(51.6 \%)$ & $121(50.2 \%)$ & 0.52 \\
\hline \multicolumn{5}{|l|}{ Comorbidities } \\
\hline $\mathrm{CHF}$ & $1(1.7 \%)$ & $23(12.6 \%)$ & $24(10.0 \%)$ & 0.02 \\
\hline Malignancy & $8(13.6 \%)$ & $32(17.6 \%)$ & $40(16.6 \%)$ & 0.60 \\
\hline OAD & $13(22.0 \%)$ & $34(18.7 \%)$ & $47(19.5 \%)$ & 0.70 \\
\hline History of smoking & $26(44.1 \%)$ & 38 (20.9\%) & $64(26.6 \%)$ & $<0.001$ \\
\hline \multicolumn{5}{|l|}{ Vital signs } \\
\hline Tmax in 1 week of PE $\left({ }^{\circ} \mathrm{F}\right)( \pm \mathrm{SD})$ & $101.576( \pm 0.911)$ & $98.719( \pm 0.801)$ & $99.419(1.483)$ & $<0.001$ \\
\hline Heart rate (beats/min) $( \pm S D)$ & $101.136( \pm 24.449)$ & $90.199( \pm 20.138)$ & $92.888(21.743)$ & $<0.001$ \\
\hline $\operatorname{MAP}(\mathrm{mm} \mathrm{Hg})( \pm \mathrm{SD})$ & $84.879( \pm 16.965)$ & $88.456( \pm 16.572)$ & $87.592(16.703)$ & 0.15 \\
\hline Systolic BP (mm Hg) $( \pm \mathrm{SD})$ & $115.932( \pm 24.143)$ & $123.720( \pm 19.685)$ & $121.813(21.080)$ & 0.01 \\
\hline Oxygen saturation (\%)(SD) & $95.724( \pm 5.742)$ & $96.808( \pm 3.900)$ & $96.535(4.448)$ & 0.10 \\
\hline \multicolumn{5}{|l|}{ PE relevant data } \\
\hline Simple PE & $26(44.1 \%)$ & $115(63.2 \%)$ & $141(58.5 \%)$ & 0.01 \\
\hline Massive and submassive PE & $33(55.9 \%)$ & 67 (36.8\%) & $100(41.5 \%)$ & 0.01 \\
\hline Use of TPA & $4(6.8 \%)$ & $2(1.1 \%)$ & $6(2.5 \%)$ & 0.05 \\
\hline Use of anticoagulation & $57(96.6 \%)$ & $164(90.1 \%)$ & $221(91.7 \%)$ & 0.19 \\
\hline Laterality (Bilateral) & $30(50.8 \%)$ & $76(41.8 \%)$ & $106(44.0 \%)$ & 0.28 \\
\hline Pulmonary infarct & $5(8.5 \%)$ & $4(2.2 \%)$ & $9(3.7 \%)$ & 0.07 \\
\hline DVT & $15 / 45(33.3 \%)$ & 23/132 (17.4\%) & $38 / 177(21.5 \%)$ & 0.03 \\
\hline RVSP n ( \pm SD) & $42.370( \pm 13.920)$ & $39.411( \pm 13.586)$ & $40.079(13.682)$ & 0.20 \\
\hline \multicolumn{5}{|l|}{ Infection data } \\
\hline Leucocyte count k/ul ( $(\mathrm{SD})$ & $10.976(5.848)$ & $8.797(5.013)$ & $9.330(5.301)$ & 0.006 \\
\hline Positive blood culture within 1 week of PE & $1(1.9 \%)$ & $1(0.7 \%)$ & $2(1.0 \%)$ & 1.000 \\
\hline Urine culture positive within 1 week of PE & $3(6.2 \%)$ & $9(7.3 \%)$ & $12(7.0 \%)$ & 1.000 \\
\hline Antibiotics given within 1 week of PE & $37(62.7 \%)$ & 49 (26.9\%) & $86(35.7 \%)$ & $<0.001$ \\
\hline
\end{tabular}

BP, blood pressure; CHF, congestive heart failure; DVT, deep venous thrombosis; MAP, mean arterial pressure; OAD, obstructive airway disease; PE, pulmonary embolism;RVSP, right ventricular systolic pressure; TPA, tissue plasminogen activator; Tmax, maximum temperature.

of smoking (44.1\% vs $20.9 \%, \mathrm{p}<0.001)$ and lower incidence of $\mathrm{CHF}(1.7 \%$ vs $12.6 \%$, $\mathrm{p}=0.029)$.

Table 2 indicates the outcomes stratified by fever status. The percentage of patients who died, need for mechanical ventilation and ICU admission were higher for patients with fever compared with patients without fever (mortality: $22.0 \%$ vs $10.4 \%$, $\mathrm{p}=0.039$; need for mechanical ventilation: $30.5 \%$ vs $6.6 \%, \mathrm{p}<0.001$; ICU admission: $69.5 \%$ vs $35.7 \%, \mathrm{p}<0.001)$. The LOS was higher for patients with fever compared with patients without fever (19.80 vs $12.20, \mathrm{p}<0.001)$.

Table 3 indicates results from regression analyses. After controlling confounders, the OR for requiring mechanical ventilation was 4.6 (CI 1.71 to 12.88). The OR of

Table 2 Outcome data

\begin{tabular}{|c|c|c|c|c|}
\hline Outcome variable & PE with fever ( $N=59)$ & $\begin{array}{l}\text { PE without fever } \\
(\mathrm{N}=182)\end{array}$ & Total=241 & $P$ values \\
\hline Need for mechanical ventilation & $18(30.5 \%)$ & $12(6.6 \%)$ & $30(12.4 \%)$ & $<0.001$ \\
\hline ICU admission & $41(69.5 \%)$ & $45(24.7 \%)$ & $86(35.7 \%)$ & $<0.001$ \\
\hline Length of hospital stay (days) $( \pm S D)$ & $19.797( \pm 18.391)$ & $12.203( \pm 12.353)$ & $14.062( \pm 14.406)$ & $<0.001$ \\
\hline Mortality & $13(22.0 \%)$ & $19(10.4 \%)$ & $32(13.3 \%)$ & 0.03 \\
\hline
\end{tabular}

ICU, intensive care unit; PE, pulmonary embolism. 


\begin{tabular}{llllc}
\hline Table 3 & Multivariable logistic regression results & & & \\
\hline & Outcome variable & Risk factor: fever status & OR (95\% Cl) & P values \\
\hline Model 1 & Death & $\begin{array}{l}\text { No fever } \\
\text { Fever }\end{array}$ & $1.92(0.76$ to 4.75$)$ & 0.16 \\
Model 2 & Needed mechanical ventilation & $\begin{array}{l}\text { No fever } \\
\text { Fever }\end{array}$ & $4.6(1.71$ to 12.88$)$ & 0.003 \\
Model 3 & ICU admission & $\begin{array}{l}\text { No fever } \\
\text { Fever }\end{array}$ & $5.74(2.73$ to 12.57$)$ & $<0.001$ \\
Model 4 & Hospital length of stay & $\begin{array}{l}\text { No fever } \\
\text { Fever }\end{array}$ & & 0.004 \\
\hline
\end{tabular}

ICU, intensive care unit.

needing ICU was 5.74 (CI 2.73 to 12.57). Having fever was associated with 6.62 (CI 2.11 to 11.14) days longer LOS in the hospital.

\section{DISCUSSION}

In this 10-year single-centre study of all hospitalised patients with newly diagnosed acute $\mathrm{PE}$, we showed that presence of fever was associated with higher likelihood of underlying massive and submassive and DVT. The need for mechanical ventilation, hospital LOS and ICU admission was also higher in patients with acute $\mathrm{PE}$ and fever. Patients with acute PE and fever also had a higher mortality in a univariate analysis. To the extent of our knowledge, our study is probably the first to extensively study this clinical and prognostic correlation of PE and fever while accounting for underlying infections.

Fever has been reported as one of the key symptoms of PE along with cough, chest pain and dyspnoea with majority of the data emphasising its association with low grade fever. ${ }^{12}{ }^{18}$ Presence of high-grade fever in the setting of thromboembolism is not supported by evidence and identification of another source has been suggested. ${ }^{18}$ In the recent data, low-grade fever in PE is detected in the range of $6 \%-33 \%$ and high-grade fever is reported variably from $3 \%$ to $14 \% .^{12-14}$ In our study, $24.5 \%$ population manifested fever with average peak temperature observed was $38.6^{\circ} \mathrm{C}\left(101.5^{\circ} \mathrm{F}\right)$ within 1 week of $\mathrm{PE}$ diagnosis. Comparably in a study by Murray et al, 18 out of 19 subjects with PE were afebrile after 1 week of diagnosis. ${ }^{14}$ The most common accompanying symptom, besides fever, manifested in our PE population was chest pain and shortness of breath which is a similar finding observed by Korkturk et al in a study of 39 patients with PE and fever. ${ }^{17}$ Identical description of symptoms including dyspnoea and restlessness was reported by Hodgosn et al in patients with $\mathrm{PE}$ and pulmonary infarction secondary to large or recurrent small emboli causing obstruction of pulmonary circulation. ${ }^{19}$

Fever that persisted beyond 1 week of initiation of anticoagulation was not attributed to PE itself and thus other causes should be evaluated. ${ }^{18}{ }^{19}$ Several aetiologies have been proposed for persistent fever after 1 week of
PE diagnosis including pulmonary infarct, superimposed infection, drug fever and Dressler like phenomenon. ${ }^{20-22}$ Stein $e t a l^{20}$ noted fever in 39 out of 267 patients with pulmonary infarction and haemorrhage and found more evidence of DVT in patients with PE and otherwise unexplained fever which was also observed in our study. Further, several patterns of PE-related fever were described based on duration including intermittent, sustained or hectic type ${ }^{20-22}$ though intermittent and sustained type were noted more in our study group. In one of the studies on complicated $\mathrm{PE},{ }^{21}$ fever was observed in $26 \%$ of heparin and urokinase-treated patients (41 out of 158). In our study, we identified that fever was more likely in patients with massive and submassive PE compared with those with simple PE (55.9\% vs $36.8 \%)$ and we did not find any statistical difference in terms of pulmonary infarcts between the two groups.

The pathophysiology of fever in PE has not been clearly understood. ${ }^{19} 2324$ Several models for its mechanism have been mentioned in literature but none is supported by the evidence. ${ }^{25-27}$ The pyrogenic model described the production of inflammation cascade associated with tissue necrosis, vascular irritation and atelectasis that leads to rise in body temperature. ${ }^{1516}$ This results in an elevated leucocyte count in the early part of disease course which was also observed in our study. ${ }^{17}$ Another mechanism explained by Jerjes and his colleagues ${ }^{22}$ is Dressler-like syndrome, caused by immune reactivity of serosa (pleura and pericardium) secondary to vascular remodelling, reported in $4 \%$ of patients with PE. This mechanism can result in pleural effusion, fever, anaemia and leucocytosis. Our study highlighted a noteworthy association of fever and higher incidence of massive/ submassive PE and DVT in patients with acute PE.

In majority of the studies performed to identify the relationship of PE and fever, exclusion of other causes of fever was not well illustrated and thus its association with PE remained debatable. ${ }^{25-27}$ Our study is noteworthy in a way that we included microbiological data and clinical decision making to identify individuals in whom fever could be attributed to PE with more certainty and excluded those where we could not establish this relation 
with certainty. The antibiotic use was higher in patients with $\mathrm{PE}$ and fever. The common reasons recognised in the literature for initiation of antibiotics in patients with $\mathrm{PE}$ are leucocytosis, infiltrates on X-ray imaging or clinical symptoms that may suggest underlying pneumonia which are probably also true for our study cohort. ${ }^{17-19}$

The data regarding outcome of individuals with PE and fever are very limited. ${ }^{252628-33}$ Our study is striking in this regard that we investigated the morbidity and mortality data for this subset of population. Watanakunakorn ${ }^{25}$ reported that nearly half of the patients with $\mathrm{PE}$ with high-grade fever in his study died. This study was a series of seven patients with limited microbiological data evaluation. Additionally, outcome and patient characteristics were not well elaborated. Calvo-Romero $e t a l^{26}$ reported in hospital mortality of $7 \%$ in patients with $\mathrm{PE}$ and fever. Although the study included majority of the patients with $\mathrm{PE}$ diagnosed by various imaging modalities; however, it is limited by the fact that investigators favoured pneumonia as the cause of fever and had not considered other sources of infection into account. Furthermore, outcome data in this study including morbidity and mortality were similar in all groups. In a 4-year prospective study on predictive factors of PE, Bahloul $e t a l^{33}$ studied outcome data in patients with PE irrespective of occurrence of fever in patients who were critically ill. They reported mean length of hospital stay (LOS) of 25 days, mean ICU stay of 20.2 days and the in-hospital mortality rate of $52.9 \%$. Even though the study included clinical characteristics, risk factors and outcomes in acute patients with PE, the authors did not signify association of fever in such patients and its clinical impact on patient outcomes. In our study, mean LOS for PE and fever was 14 days, with higher likelihood of ICU admission in fever group $(69.5 \%$ vs $24.7 \%)$. We also found higher mortality in patients with $\mathrm{PE}$ and fever (22\% vs $10.4 \%$ ) along with higher need for mechanical ventilation ( $30 \%$ vs $6.6 \%)$.

There are several limitations of our study. First, we used a retrospective design where we were limited to the information obtained by the medical records and not direct patient contact. Second, we included patients from a single centre, which cares for an inner-city population with specific demographic characteristics. Third, our study group is relatively small compared with some large PE study which can affect study power. However, we believe that even with small patient population, our study was able to answer some interesting clinical questions. Fourth, we retrospectively reviewed all septic workup data in patients admitted with acute PE and fever and excluded all those patients with underlying infections. This could introduce selection bias. To address that bias, two study investigators (MS and MA) independently reviewed the patient charts and excluded patients where underlying cause of fever may be attributed to non-PE source. Fifth, we did not include any patients who had fever more than 1 week from diagnosis of PE. This was done in view of prior data that stated that fever beyond 1 week is less likely to be associated with PE. ${ }^{11} 121416$
Sixth, since it is a 10 -year study, advancement in medical practice might be a confounding factor which may have affected the study results. Finally, we did not include ICU severity scoring system (APACHE or SOFA scores) to estimate its contribution to mortality and LOS. Despite the limitations, our study highlights the potential association of fever with clot burden in acute PE that has both clinical and prognostic implications in care of these patients.

\section{CONCLUSION}

Fever in patients with acute PE is associated with distinct clinical and prognostic parameters. Patients with acute $\mathrm{PE}$ and fever are more likely to have massive or submassive PE and DVT. Presence of fever was also associated with higher need mechanical ventilation, ICU admission and longer hospital LOS. Larger prospective studies are needed to validate our findings.

Contributors MS, MA and AZ wrote the first draft of the manuscript and reviewed the data for its integrity. DHS, AA, NM, MS and MA did the data collection. AZ performed the statistical analysis for the study. All authors reviewed, edited and approved the final draft of this manuscript. MA is guarantor of this paper and take full responsibility for the integrity of the work as a whole, from inception to publication.

Funding The authors have not declared a specific grant for this research from any funding agency in the public, commercial or not-for-profit sectors.

Competing interests None declared.

Patient consent Not required.

Ethics approval BronxCare Hospital Center, IRB\#01111803.

Provenance and peer review Not commissioned; externally peer reviewed.

Data statement Data available upon request

Open access This is an open access article distributed in accordance with the Creative Commons Attribution Non Commercial (CC BY-NC 4.0) license, which permits others to distribute, remix, adapt, build upon this work non-commercially, and license their derivative works on different terms, provided the original work is properly cited, appropriate credit is given, any changes made indicated, and the use is non-commercial. See: http://creativecommons.org/licenses/by-nc/4.0

\section{REFERENCES}

1. Giuntini C, Di Ricco G, Marini C, et al. Pulmonary embolism: epidemiology. Chest 1995;107(1 Suppl):3S-9.

2. Goldhaber SZ, Visani L, De Rosa M. Acute pulmonary embolism: clinical outcomes in the International Cooperative Pulmonary Embolism Registry (ICOPER). Lancet 1999;353:1386-9.

3. Anderson FA, Zayaruzny M, Heit JA, et al. Estimated annual numbers of US acute-care hospital patients at risk for venous thromboembolism. Am J Hematol 2007;82:777-82.

4. Cushman M, Tsai A, Heckbert SR. Incidence rates, case fatality, and recurrence rates of deep vein thrombosis and pulmonary embolus: the Longitudinal Investigation of Thromboembolism Etiology (LITE). Thromb Haemost 2001;86.

5. Anderson FA, Wheeler HB, Goldberg RJ, et al. A population-based perspective of the hospital incidence and case-fatality rates of deep vein thrombosis and pulmonary embolism. The Worcester DVT Study. Arch Intern Med 1991;151:933-8.

6. Mamlouk MD, vanSonnenberg E, Gosalia R, et al. Pulmonary embolism at CT angiography: implications for appropriateness, cost, and radiation exposure in 2003 patients. Radiology 2010;256:625-32.

7. Dobesh PP. Economic burden of venous thromboembolism in hospitalized patients. Pharmacotherapy 2009;29:943-53.

8. Silverstein MD, Heit JA, Mohr DN, et al. Trends in the incidence of deep vein thrombosis and pulmonary embolism: a 25-year population-based study. Arch Intern Med 1998;158:585-93.

9. MacDougall DA, Feliu AL, Boccuzzi SJ, et al. Economic burden of deep-vein thrombosis, pulmonary embolism, and post-thrombotic syndrome. Am J Health Syst Pharm 2006;63(20 Suppl 6):S5-S15. 
10. Miniati M, Bottai M, Monti S, et al. Simple and accurate prediction of the clinical probability of pulmonary embolism. Am J Respir Crit Care Med 2008;178:290-4.

11. Stein PD, Terrin ML, Hales CA, et al. Clinical, laboratory, roentgenographic, and electrocardiographic findings in patients with acute pulmonary embolism and no pre-existing cardiac or pulmonary disease. Chest 1991;100:598-603.

12. Stein PD, Beemath A, Matta F, et al. Clinical characteristics of patients with acute pulmonary embolism: data from PIOPED II. Am J Med 2007;120:871-9.

13. Miniati M, Pistolesi M, Marini $C$, et al. Value of perfusion lung scan in the diagnosis of pulmonary embolism: results of the Prospective Investigative Study of Acute Pulmonary Embolism Diagnosis (PISAPED). Am J Respir Crit Care Med 1996:154:1387-93.

14. Murray HW, Ellis GC, Blumenthal DS, et al. Fever and pulmonary thromboembolism. Am J Med 1979;67:232-5.

15. Israel HL, Goldstein $\mathrm{F}$. The varied clinical manifestations of pulmonary embolism. Ann Intern Med 1957;47:202-26.

16. Miniati M, Cenci C, Monti S, et al. Clinical presentation of acute pulmonary embolism: survey of 800 cases. PLoS One 2012;7:e30891.

17. Kokturk N, Demir N, Oguzulgen IK, et al. Fever in pulmonary embolism. Blood Coagul Fibrinolysis 2005;16:341-7.

18. Stein PD, Henry JW. Prevalence of acute pulmonary embolism among patients in a general hospital and at autopsy. Chest 1995;108:978-81.

19. Hodgson CH. Pulmonary embolism and. Dis Chest 1965;47:577-88.

20. Wenger NK, Stein PD, Willis PW. Massive acute pulmonary embolism. The deceivingly nonspecific manifestations. JAMA 1972;220:843-4.

21. Study NC. The urokinase pulmonary embolism trial. Circulation 1973;47(/48):II-81--80.
22. Jerjes-Sanchez C, Ibarra-Perez C, Ramirez-Rivera A, et al. Dresslerlike syndrome after pulmonary embolism and infarction. Chest 1987:92:115-7.

23. Nucifora G, Badano L, Hysko F, et al. Pulmonary embolism and fever: when should right-sided infective endocarditis be considered? Circulation 2007;115:e173-e176.

24. Afzal A, Noor HA, Gill SA, et al. Leukocytosis in acute pulmonary embolism. Chest 1999;115:1329-32.

25. Watanakunakorn C, Hayek F. High fever (greater than 39 degrees C) as a clinical manifestation of pulmonary embolism. Postgrad Med $J$ 1987;63:951-3.

26. Calvo-Romero JM, Lima-Rodríguez EM, Pérez-Miranda M, et al. Low-grade and high-grade fever at presentation of acute pulmonary embolism. Blood Coagul Fibrinolysis 2004;15:331-3.

27. Goldhaber SZ. Pulmonary embolism. N Engl J Med 1998;339:93-104.

28. Fedullo PF, Tapson VF. Clinical practice. The evaluation of suspected pulmonary embolism. N Engl J Med 2003;349:1247-56.

29. Sharma GV, Sasahara AA, McIntyre KM. Pulmonary embolism: the great imitator. Dis Mon 1976;22:1-38.

30. Mclntyre KM, Sasahara AA. Sharma GURK: Pulmonary thrornboembolism: current concepts. Adv Intern Med 1972;18: 19C.

31. Saad M, Adrish M, Adrish M. A rare case report of a saddle pulmonary embolism presenting with high grade fevers, responsive to anticoagulation. Medicine 2018;97:e0002.

32. Horlander KT, Mannino DM, Leeper KV. Pulmonary embolism mortality in the United States, 1979-1998: an analysis using multiplecause mortality data. Arch Intern Med 2003;163:1711-7.

33. Bahloul $\mathrm{M}$, Chaari $\mathrm{A}$, Kallel $\mathrm{H}$, et al. Pulmonary embolism in intensive care unit: Predictive factors, clinical manifestations and outcome. Ann Thorac Med 2010;5:97-103. 